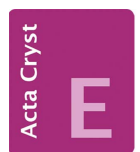

CRYSTALLOGRAPHIC COMMUNICATIONS

ISSN 2056-9890

Received 1 August 2017

Accepted 3 October 2017

Edited by M. Weil, Vienna University of Technology, Austria

Keywords: crystal structure; iodide bridge; C,Ncyclopalladated complex.

CCDC reference: 1577834

Supporting information: this article has supporting information at journals.iucr.org/e

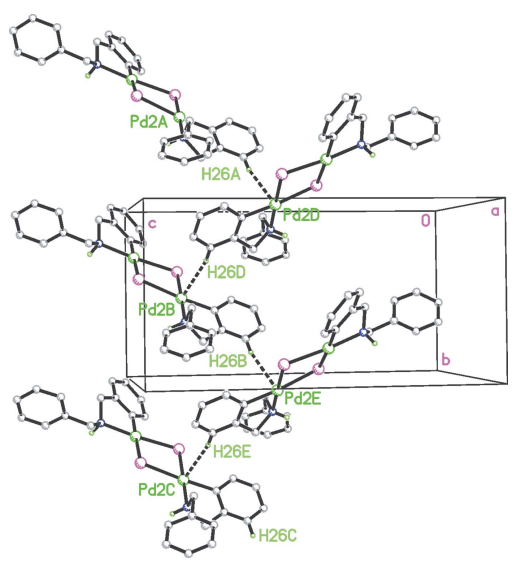

OPEN $\odot$ ACCESS

\section{Synthesis and crystal structure of $\left[\mathrm{Pd}\left\{\mathrm{C}_{6} \mathrm{H}_{4}\left(\mathrm{CH}_{2} \mathrm{NHCH}_{2} \mathrm{Ph}\right)-2-\kappa^{2} \mathrm{C}, \mathrm{N}\right\}(\mu-\mathrm{I})\right]_{2}$}

\author{
Delia Bautista $^{\mathrm{a} *}$ and Sergio J. Benitez-Benitez ${ }^{\mathrm{b}}$
}

${ }^{a}$ SAI, Universidad de Murcia, Murcia 30100, Spain, and ${ }^{\mathbf{b}}$ Departamento Química Inorgánica, Universidad de Murcia, Murcia 30071, Spain. *Correspondence e-mail: dbc@um.es

The binuclear title complex, di- $\mu$-iodido-bis $(\{2-[($ benzylamino- $\kappa N)$ methyl]phenyl- $\kappa C^{1}$ \}palladium(II)), $\left[\mathrm{Pd}_{2} \mathrm{I}_{2}\left(\mathrm{C}_{14} \mathrm{H}_{14} \mathrm{~N}\right)_{2}\right]$, was prepared by reaction of $\left[\mathrm{Pd}\left\{\mathrm{C}_{6} \mathrm{H}_{4}\left(\mathrm{CH}_{2} \mathrm{NHCH}_{2} \mathrm{Ph}\right)-2\right\}(\mu-\mathrm{OAc})\right]_{2}$ with $\mathrm{NaI}$. It crystallizes with one discrete molecule in the asymmetric unit. The molecule presents an iodidebridged dimeric structure with a cisoid arrangement with respect to the $C, N$ cyclopalladated ligands. Both $\mathrm{Pd}^{\mathrm{II}}$ atoms have a slightly distorted square-planar coordination environment. Weak intermolecular contacts of the type $\mathrm{C}-$ $\mathrm{H}$..Pd seem to have a significant influence on the arrangement of the molecules along the $b$ axis in the crystal.

\section{Chemical context}

Cyclopalladated complexes (Dupont et al., 2005) have important applications in homogeneous catalysis (Bravo et al., 2002), as chiral resolving agents (Gugger et al., 2008), drugs (Cutillas et al., 2013), or new materials (Jayabharathi et al., 2011).

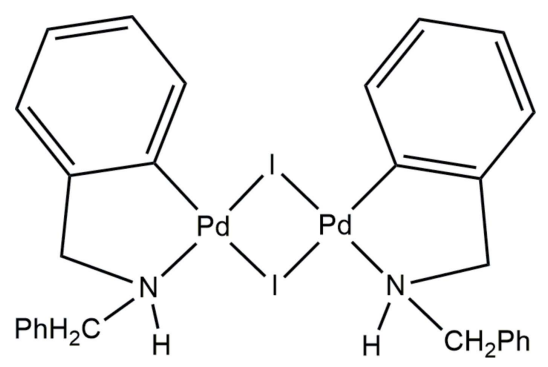

Over the past few years, our group has been interested in the synthesis, reactivity and applications in organic synthesis of orthopalladated derivatives of dibenzylamine. We have reported the first general method for the cyclopalladation of primary and secondary amines by using $\operatorname{Pd}(\mathrm{OAc})_{2}$. The acetato-bridged complexes were transformed into the corresponding halido-bridged complexes by anion metathesis reactions, which were used for further reactivity studies (Vicente $e t$ al., 1997).

Herein we report the synthesis and crystal structure of a iodido-bridged complex $\left[\mathrm{Pd}\left\{\mathrm{C}_{6} \mathrm{H}_{4}\left(\mathrm{CH}_{2} \mathrm{NHCH}_{2} \mathrm{Ph}\right)-2\right\}(\mu-\mathrm{I})\right]_{2}$. This is a rare example of a cyclopalladated complex containing bridging iodido ligands and one of the few $\hat{\mathrm{C} N}$-cyclopalladated iodido-bridged complexes characterized by $\mathrm{X}$-ray diffraction.

\section{Structural commentary}

The complex crystallizes in the centrosymmetric monoclinic space group $P 2_{1} / n$ with one molecule in the asymmetric unit. 
Table 1

Selected torsion angles $\left({ }^{\circ}\right)$.

\begin{tabular}{lrll}
\hline $\mathrm{C} 2-\mathrm{C} 1-\mathrm{C} 7-\mathrm{N} 1$ & $23.5(6)$ & $\mathrm{C} 22-\mathrm{C} 21-\mathrm{C} 27-\mathrm{N} 2$ & $29.2(6)$ \\
$\mathrm{C} 7-\mathrm{N} 1-\mathrm{C} 8-\mathrm{C} 11$ & $-54.9(7)$ & $\mathrm{C} 27-\mathrm{N} 2-\mathrm{C} 28-\mathrm{C} 31$ & $67.4(5)$ \\
\hline
\end{tabular}

The molecular structure can be described as a nearly planar dipalladium subunit of the type $(\mathrm{C}-\mathrm{N}) \mathrm{Pd}(\mu-\mathrm{I})_{2} \mathrm{Pd}(\mathrm{C}-\mathrm{N})$ (Fig. 1). Both palladium atoms adopt a slightly distorted square-planar coordination environment, the mean deviations of the $\mathrm{Pd}-\mathrm{N}-\mathrm{C}-\mathrm{I}-\mathrm{I}$ planes being larger for $\mathrm{Pd} 2(0.0868 \AA)$ than for $\operatorname{Pd} 1(0.0301 \AA)$. The highest deviation from the average coordination plane occurs for C22 (0.1261 $\AA$ ). The more distorted square-planar geometry of $\mathrm{Pd} 2$ is further evidenced by the smaller dihedral angle between the planes $\mathrm{N} 1-\mathrm{Pd} 1-\mathrm{C} 2$ and $\mathrm{I} 1-\mathrm{Pd} 1-\mathrm{I} 2$ [5.53 (16) $\left.^{\circ}\right]$ compared to that of $\mathrm{N} 2-\mathrm{Pd} 2-\mathrm{C} 22$ and $\mathrm{I} 1-\mathrm{Pd} 2-\mathrm{I} 2$ [8.29 (16) ${ }^{\circ}$. The structural differences around both $\mathrm{Pd}^{\mathrm{II}}$ atoms are consistent with the presence of two $\mathrm{N}-\mathrm{H}$ stretching bands at 3261 and $3201 \mathrm{~cm}^{-1}$ in the infrared spectrum of the solid.

In contrast to the unsymmetrical dimers with a cisoid arrangement of the ligands observed in the title compound $\left[\mathrm{Pd}\left\{\mathrm{C}_{6} \mathrm{H}_{4}\left(\mathrm{CH}_{2} \mathrm{NHCH}_{2} \mathrm{Ph}\right){ }_{2}\right\}(\mu-\mathrm{I})\right]_{2}$, the dibromido analogue $\left[\mathrm{Pd}\left\{\mathrm{C}_{6} \mathrm{H}_{4}\left(\mathrm{CH}_{2} \mathrm{NHCH}_{2} \mathrm{Ph}\right)-2\right\}(\mu-\mathrm{Br})\right]_{2}$ (Vicente et al., 1999) shows a centrosymmetric dimer with a transoid disposition of the chelating ligands involving the amino groups.

Owing to the cisoid arrangement of the C,N-cyclopalladated ligands, one of the iodine atoms of the $\operatorname{Pd}_{2} \mathrm{I}_{2}$ unit is trans to two carbon atoms (I1) whereas the other is trans to two nitrogen atoms (I2). Consequently, the $\mathrm{Pd}-\mathrm{I}$ bond lengths of the I atoms trans to $\mathrm{N}$ [2.5959 (5) and 2.5801 (4) $\AA$ ] are shorter than those of the I atoms trans to C [2.7504 (5) and 2.7030 (5) $\AA$ ] because of the greater trans influence of the aryl ligands compared to that of the amino ligands. Similar values for these bond lengths and also for the C-Pd [1.986 (5), 1.991 (4) ̊] and N-Pd [2.104 (4), 2.809 (4) A] bond lengths

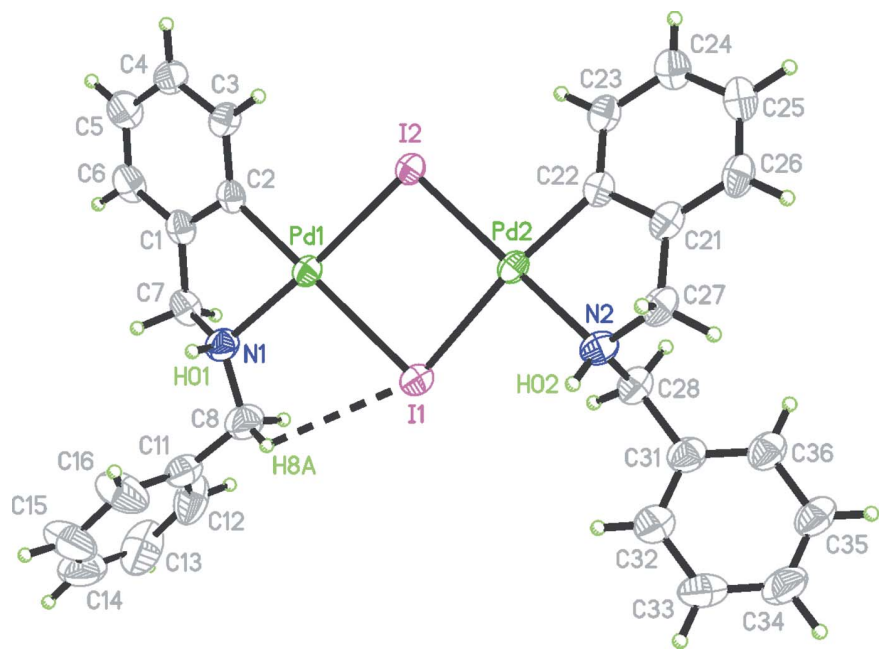

Figure 1

The molecular structure of the title complex, with displacement ellipsoids at the $50 \%$ probability level. The black dashed line indicates the intramolecular $\mathrm{C}-\mathrm{H} \cdots \mathrm{I}$ hydrogen bond (see Table 2 for numerical details).
Table 2

Hydrogen-bond geometry $\left(\AA{ }^{\circ}\right)$.

\begin{tabular}{lllll}
\hline$D-\mathrm{H} \cdots A$ & $D-\mathrm{H}$ & $\mathrm{H} \cdots A$ & $D \cdots A$ & $D-\mathrm{H} \cdots A$ \\
\hline $\mathrm{C} 8-\mathrm{H} 8 A \cdots \mathrm{I} 1$ & 0.99 & 2.94 & $3.444(6)$ & 113 \\
\hline
\end{tabular}

have been found in the five structures of iodido-bridged cyclopalladated complexes reported so far (see Database survey). Selected torsion angles are collated in Table 1.

One of the methylenic hydrogen atoms of the cyclopalladated dibenzylamine moiety coordinating to Pd1 participates in the formation of a non-classical intramolecular $\mathrm{C}-$ H...I hydrogen bond (Fig. 1, Table 2).

\section{Supramolecular features}

There are no hydrogen-bonding interactions involving the two NH groups. The most remarkable intermolecular interaction observed in the crystal structure is a weak hydrogen bond between the arylic hydrogen placed in position 3 of the phenylene ring attached to $\mathrm{Pd} 2$ (H16) and the $\mathrm{Pd} 2$ atom of the adjacent molecule. This interaction gives rise to the formation of a chain arrangement of molecules along the $b$ axis (Fig. 2). Although the $\mathrm{Pd}-\mathrm{H}$ bond length [2.760 (2) $\AA$ ] is slightly shorter than the sum of the van der Waals radii of $\mathrm{Pd}$ and $\mathrm{H}$ (2.83 $\AA$ ) (Bondi, 1964), it seems to direct the arrangement of the molecules in the crystal structure. In this context it is interesting to compare the arrangement of the molecules in

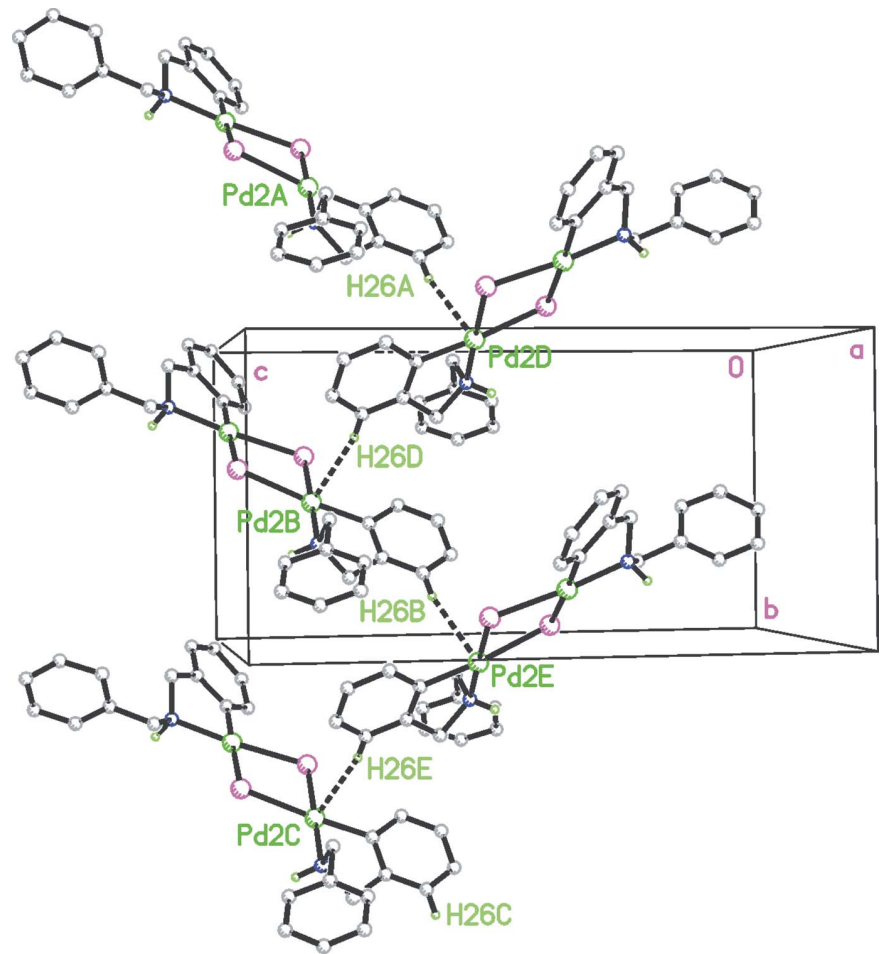

Figure 2

A view of the molecular packing of the title compound. Dotted lines indicate $\mathrm{C}-\mathrm{H} \cdots \mathrm{Pd}$ contacts. $\mathrm{H}$ atoms not involved in the interactions have been omitted for clarity. 
this complex with that of the dibromido analogue $\left[\mathrm{Pd}\left\{\mathrm{C}_{6} \mathrm{H}_{4}\left(\mathrm{CH}_{2} \mathrm{NHCH}_{2} \mathrm{Ph}\right){ }_{2}\right\}(\mu-\mathrm{Br})\right]_{2}$ (Vicente et al., 1999), which is formed by stacking of nearly co-planar complex palladium dimers, where the empty space is filled by solvent molecules $\left(\mathrm{CH}_{2} \mathrm{Cl}_{2}\right)$. Such a disposition appears to be normal in dimeric halido-bridging cyclometalated complexes of $d^{8}$ elements (Aullón et al., 1998) and hence contrasts with the unusual structure observed in the title compound $\left[\mathrm{Pd}\left\{\mathrm{C}_{6} \mathrm{H}_{4}\left(\mathrm{CH}_{2} \mathrm{NHCH}_{2} \mathrm{Ph}\right)-2\right\}(\mu-\mathrm{I})\right]_{2}$.

\section{Database survey}

A search in the Cambridge Structural Database (Groom et al., 2016) gave only six reports of binuclear iodido-bridged orthopalladated complexes with different bidentate $\mathrm{C}-\mathrm{N}$ ligands: $N, N$-dimethylbenzylamine (Gül \& Nelson, 2000), azobenzene derivatives (Ghedini et al., 1999; Crispini et al., 1993), imines (Praefcke et al., 1995) and ferrocenyloxazoline derivatives (Donde \& Overman, 1999; Anderson et al., 2005), with the following bond lengths ranges: Pd-I: 2.591 (3)2.581 (5) $\AA$ (trans to N), 2.724 (4)- 2.694 (5) $\AA$ (trans to C);

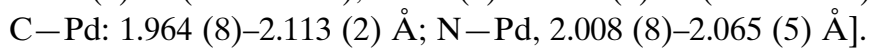

\section{Synthesis and crystallization}

To a suspension of the complex $\left[\mathrm{Pd}\left\{\mathrm{C}_{6} \mathrm{H}_{4}\left(\mathrm{CH}_{2} \mathrm{NHCH}_{2} \mathrm{Ph}\right)-2\right\}\right.$ $(\mu \text {-OAc) }]_{2}$ (Vicente et al., 1999) (800 mg, $\left.1.106 \mathrm{mmol}\right)$ in acetone $(30 \mathrm{ml})$ solid $\mathrm{NaI}(1000 \mathrm{mg}, 6.022 \mathrm{mmol})$ was added and the resulting mixture was stirred for $3 \mathrm{~h}$. The solution was filtered through a plug of $\mathrm{MgSO}_{4}$, and the filtrate was concentrated to $\mathrm{ca} 5 \mathrm{ml}$. Diethyl ether was added $(25 \mathrm{ml})$, the solvent was partially removed (to $c a 5 \mathrm{ml}$ ), and $n$-pentane was added $(25 \mathrm{ml})$ to precipitate the title complex as an orange solid, which was collected and air-dried. Single crystals of the compound suitable for X-ray analysis were obtained by slow diffusion of $n$-pentane into a solution of the compound in $\mathrm{CHCl}_{3}$ at room temperature. Yield $845 \mathrm{mg}, 0.983 \mathrm{mmol}, 89 \%$. Analysis calculated for $\mathrm{C}_{28} \mathrm{H}_{28} \mathrm{I}_{2} \mathrm{~N}_{2} \mathrm{Pd}_{2}$ (859.2): C, 39.11; H, 3.26; N, 3.26. Found: C, 38.80; H, 3.21; N, 3.21. IR (Nujol, $\left.\mathrm{cm}^{-1}\right): v(\mathrm{~N}-\mathrm{H})=3261,3201 .{ }^{1} \mathrm{H} \mathrm{NMR}\left(\mathrm{CDCl}_{3}, 400 \mathrm{MHz}\right): d=$ 3.83-3.95 ( $\left.m, 2 \mathrm{H}, \mathrm{CH}_{2}\right), 4.18(s, b, 1 \mathrm{H}, \mathrm{NH}), 4.23-4.29(m, 1 \mathrm{H}$, $\left.\mathrm{CH}_{2}\right), 4.65\left(d, b, 1 \mathrm{H}, \mathrm{CH}_{2},{ }^{2} J_{\mathrm{HH}}=12.9 \mathrm{~Hz}\right), 6.83-6.87(m, 1 \mathrm{H}$, $\left.\mathrm{CH}, \mathrm{C}_{6} \mathrm{H}_{4}\right), 6.92-7.00\left(m, 2 \mathrm{H}, \mathrm{CH}, \mathrm{C}_{6} \mathrm{H}_{4}\right), 7.32-7.41(m, 5 \mathrm{H}$, $\mathrm{Ph}), 7.67\left(s, b, 1 \mathrm{H}, \mathrm{C}_{6} \mathrm{H}_{4},{ }^{3} J_{\mathrm{HH}}=7.6 \mathrm{~Hz}\right) .{ }^{13} \mathrm{C}\left\{{ }^{1} \mathrm{H}\right\} \mathrm{NMR}$ $\left(\mathrm{CDCl}_{3}, 75 \mathrm{MHz}\right): d=57.4\left(s, \mathrm{CH}_{2}\right), 59.4\left(s, \mathrm{CH}_{2}\right), 122.6(s$, $\left.\mathrm{CH}, \mathrm{C}_{6} \mathrm{H}_{4}\right), 124.6\left(s, \mathrm{CH}, \mathrm{C}_{6} \mathrm{H}_{4}\right), 126.4\left(s, \mathrm{CH}, \mathrm{C}_{6} \mathrm{H}_{4}\right), 128.6(s$, $p$-CH, Ph), 129.1 ( $s, m-\mathrm{CH}, \mathrm{Ph}), 129.3(s, o-\mathrm{CH}, \mathrm{Ph}), 135.6(s$, $i$-C, $\mathrm{Ph}), 138.5\left(s, \mathrm{CH}, \mathrm{C}_{6} \mathrm{H}_{4}\right), 147.8\left(s, \mathrm{C}, \mathrm{C}_{6} \mathrm{H}_{4}\right), 150.6(s, \mathrm{C}$, $\left.\mathrm{C}_{6} \mathrm{H}_{4}\right)$.

\section{Refinement}

Crystal data, data collection and structure refinement details are summarized in Table 3. $\mathrm{C}$ and $\mathrm{N}$ atoms were subjected to DELU commands (Sheldrick, 2015), and five reflections were omitted from the final refinement due to poor agreement between measured and calculated intensities. All $\mathrm{H}$ atoms
Table 3

Experimental details.

\begin{tabular}{|c|c|}
\hline \multicolumn{2}{|l|}{ Crystal data } \\
\hline Chemical formula & {$\left[\mathrm{Pd}_{2} \mathrm{I}_{2}\left(\mathrm{C}_{14} \mathrm{H}_{14} \mathrm{~N}\right)_{2}\right]$} \\
\hline$M_{\mathrm{r}}$ & 859.12 \\
\hline Crystal system, space group & Monoclinic, $P 2_{1} / n$ \\
\hline Temperature (K) & 100 \\
\hline$a, b, c(\AA)$ & $\begin{array}{l}14.2201(12), 9.9787(7) \\
19.4205(13)\end{array}$ \\
\hline$\beta\left(^{\circ}\right)$ & $90.200(2)$ \\
\hline$V\left(\AA^{3}\right)$ & $2755.7(4)$ \\
\hline$Z$ & 4 \\
\hline Radiation type & Мо $K \alpha$ \\
\hline$\mu\left(\mathrm{mm}^{-1}\right)$ & 3.57 \\
\hline Crystal size $(\mathrm{mm})$ & $0.15 \times 0.10 \times 0.04$ \\
\hline \multicolumn{2}{|l|}{ Data collection } \\
\hline Diffractometer & Bruker D8 QUEST \\
\hline Absorption correction & $\begin{array}{l}\text { Multi-scan (SADABS; Krause et } \\
\text { al., 2015) }\end{array}$ \\
\hline$T_{\min }, T_{\max }$ & $0.788,0.928$ \\
\hline $\begin{array}{l}\text { No. of measured, independent and } \\
\text { observed }[I>2 \sigma(I)] \text { reflections }\end{array}$ & $70523,5768,5148$ \\
\hline$R_{\text {int }}$ & 0.034 \\
\hline$(\sin \theta / \lambda)_{\max }\left(\AA^{-1}\right)$ & 0.630 \\
\hline \multicolumn{2}{|l|}{ Refinement } \\
\hline$R\left[F^{2}>2 \sigma\left(F^{2}\right)\right], w R\left(F^{2}\right), S$ & $0.031,0.084,1.03$ \\
\hline No. of reflections & 5768 \\
\hline No. of parameters & 315 \\
\hline No. of restraints & 319 \\
\hline $\mathrm{H}$-atom treatment & $\begin{array}{l}\mathrm{H} \text { atoms treated by a mixture of } \\
\text { independent and constrained } \\
\text { refinement }\end{array}$ \\
\hline$\Delta \rho_{\max }, \Delta \rho_{\min }\left(\mathrm{e} \AA^{-3}\right)$ & $2.45,-0.65$ \\
\hline
\end{tabular}

Computer programs: APEX2 and SAINT (Bruker, 2013), SHELXS97 and SHELXTL (Sheldrick, 2008) and SHELXL2013 (Sheldrick, 2015).

associated with $\mathrm{C}$ atoms could be located in difference-Fourier maps. However, they were relocated at geometrically idealized positions and were allowed to ride on the parent atoms with $\mathrm{C}-\mathrm{H}=0.95 \AA$ (aromatic) and $0.99 \AA\left(\mathrm{CH}_{2}\right)$ and $U_{\text {iso }}(\mathrm{H})=$ $1.2 U_{\text {eq }}(\mathrm{C})$. Hydrogen atoms bound to $\mathrm{N}$ atoms were discernible from a difference-Fourier map and were subsequently refined with $\mathrm{N}-\mathrm{H}$ distance restraints [target value 0.87 (2) $\AA$ ].

\section{Acknowledgements}

The authors gratefully acknowledge the help of I. SauraLlamas and J. Gil-Rubio from the Universidad de Murcia.

\section{Funding information}

The Spanish Ministerio de Ciencia e Innovación (grant CTQ2011-24016, with FEDER support) is acknowledged for financial support.

\section{References}

Anderson, C. E., Donde, Y., Douglas, C. J. \& Overman, L. E. (2005). J. Org. Chem. 70, 648-657.

Aullón, G., Ujaque, G., Lledós, A., Álvarez, S. \& Alemany, P. (1998). Inorg. Chem. 37, 804-813.

Bondi, A. (1964). J. Phys. Chem. 68, 441-451.

Bravo, J., Cativiela, C., Navarro, R. \& Urriolabeitia, E. P. (2002). J. Organomet. Chem. 650, 157-172. 
Bruker (2013). APEX2 and SAINT. Bruker AXS Inc. Madison Wisconsin, USA.

Crispini, A., Ghedini, M. \& Neve, F. (1993). J. Organomet. Chem. 448, 241-245.

Cutillas, N., Yellol, G. S., de Haro, C., Vicente, C., Rodríguez, V. \& Ruiz, J. (2013). Coord. Chem. Rev. 257, 2784-2797.

Donde, Y. \& Overman, L. E. (1999). J. Am. Chem. Soc. 121, $2933-$ 2934.

Dupont, J., Consorti, C. S. \& Spencer, J. (2005). Chem. Rev. 105, $2527-$ 2571.

Ghedini, M., Pucci, D., Crispini, A., Aiello, I., Barigelletti, F., Gessi, A. \& Francescangeli, O. (1999). Appl. Organomet. Chem. 13, 565581.

Groom, C. R., Bruno, I. J., Lightfoot, M. P. \& Ward, S. C. (2016). Acta Cryst. B72, 171-179.
Gugger, P. A., Hockless, D. C., Kilah, N. L., Mayadunne, R. C. \& Wild, S. B. (2008). Tetrahedron Asymmetry, 19, 1810-1812.

Gül, N. \& Nelson, J. H. (2000). Organometallics, 19, 91-104.

Jayabharathi, J., Thanikachalam, V., Saravanan, K. \& Srinivasan, N. (2011). J. Fluoresc. 21, 507-519.

Krause, L., Herbst-Irmer, R., Sheldrick, G. M. \& Stalke, D. (2015). J. Appl. Cryst. 48, 3-10.

Praefcke, K., Diele, S., Pickardt, J., Gündogman, B., Nütz, U. \& Singer, D. (1995). Liq. Cryst. 18, 857-865.

Sheldrick, G. M. (2008). Acta Cryst. A64, 112-122.

Sheldrick, G. M. (2015). Acta Cryst. C71, 3-8.

Vicente, J., Saura-Llamas, I., Palin, M. G., Jones, P. G. \& Ramírez de Arellano, M. C. (1997). Organometallics, 16, 826-833.

Vicente, J., Saura-Llamas, I., Turpín, J., Ramírez de Arellano, M. C. \& Jones, P. G. (1999). Organometallics, 18, 2683-2693. 


\section{supporting information}

Acta Cryst. (2017). E73, 1612-1615 [https://doi.org/10.1107/S2056989017014281]

Synthesis and crystal structure of $\left[\mathrm{Pd}\left\{\mathrm{C}_{6} \mathrm{H}_{4}\left(\mathrm{CH}_{2} \mathrm{NHCH} \mathrm{H}_{2} \mathrm{Ph}\right)-2-\kappa^{2} \mathrm{C}, \mathrm{N}\right\}(\mu-\mathrm{I})\right]_{2}$

\section{Delia Bautista and Sergio J. Benitez-Benitez}

Computing details

Data collection: APEX2 (Bruker, 2013); cell refinement: SAINT (Bruker, 2013); data reduction: SAINT (Bruker, 2013); program(s) used to solve structure: SHELXS97 (Sheldrick, 2008); program(s) used to refine structure: SHELXL2013

(Sheldrick, 2015); molecular graphics: SHELXTL (Sheldrick, 2008); software used to prepare material for publication: SHELXTL (Sheldrick, 2008).

Di- $\mu$-iodido-bis(\{2-[(benzylamino- $\kappa \mathrm{N})$ methyl] phenyl- $\left.\kappa C^{1}\right\}$ palladium(II))

Crystal data

$\left[\mathrm{Pd}_{2} \mathrm{I}_{2}\left(\mathrm{C}_{14} \mathrm{H}_{14} \mathrm{~N}\right)_{2}\right]$

$M_{r}=859.12$

Monoclinic, $P 22_{1} / n$

$a=14.2201(12) \AA$

$b=9.9787(7) \AA$

$c=19.4205(13) \AA$

$\beta=90.200(2)^{\circ}$

$V=2755.7(4) \AA^{3}$

$Z=4$

Data collection

Bruker D8 QUEST

diffractometer

Radiation source: high brilliance microfocus sealed tube

Detector resolution: 10.4167 pixels $\mathrm{mm}^{-1}$

$\omega$-scans

Absorption correction: multi-scan

(SADABS; Krause et al., 2015)

$T_{\min }=0.788, T_{\max }=0.928$

Refinement

Refinement on $F^{2}$

Least-squares matrix: full

$R\left[F^{2}>2 \sigma\left(F^{2}\right)\right]=0.031$

$w R\left(F^{2}\right)=0.084$

$S=1.03$

5768 reflections

315 parameters

319 restraints
$F(000)=1632$

$D_{\mathrm{x}}=2.071 \mathrm{Mg} \mathrm{m}^{-3}$

Mo $K \alpha$ radiation, $\lambda=0.71073 \AA$

Cell parameters from 9807 reflections

$\theta=2.3-26.6^{\circ}$

$\mu=3.57 \mathrm{~mm}^{-1}$

$T=100 \mathrm{~K}$

Lath, orange

$0.15 \times 0.10 \times 0.04 \mathrm{~mm}$

70523 measured reflections

5768 independent reflections

5148 reflections with $I>2 \sigma(I)$

$R_{\text {int }}=0.034$

$\theta_{\text {max }}=26.6^{\circ}, \theta_{\min }=2.1^{\circ}$

$h=-17 \rightarrow 17$

$k=-12 \rightarrow 12$

$l=-24 \rightarrow 24$

Hydrogen site location: mixed

$\mathrm{H}$ atoms treated by a mixture of independent and constrained refinement

$w=1 /\left[\sigma^{2}\left(F_{\mathrm{o}}^{2}\right)+(0.0439 P)^{2}+10.4728 P\right]$

where $P=\left(F_{\mathrm{o}}{ }^{2}+2 F_{\mathrm{c}}{ }^{2}\right) / 3$

$(\Delta / \sigma)_{\max }=0.001$

$\Delta \rho_{\max }=2.45 \mathrm{e}^{-3}$

$\Delta \rho_{\min }=-0.65$ e $\AA^{-3}$ 


\section{Special details}

Geometry. All esds (except the esd in the dihedral angle between two 1.s. planes) are estimated using the full covariance matrix. The cell esds are taken into account individually in the estimation of esds in distances, angles and torsion angles; correlations between esds in cell parameters are only used when they are defined by crystal symmetry. An approximate (isotropic) treatment of cell esds is used for estimating esds involving l.s. planes.

Least-squares planes (x,y,z in crystal coordinates) and deviations from them (* indicates atom used to define plane) $6.1807(0.0096) \mathrm{x}-8.2633(0.0047) \mathrm{y}+6.8462(0.0164) \mathrm{z}=3.8899(0.0181)$

* $0.0632(0.0014) \mathrm{Pd} 1 * 0.0071(0.0020) \mathrm{N} 1 *-0.0439(0.0021) \mathrm{C} 2 *-0.0312(0.0014) \mathrm{I} 1 * 0.0048(0.0016) \mathrm{I} 2$

Rms deviation of fitted atoms $=0.0373$

$7.4752(0.0099) \mathrm{x}-7.3667(0.0058) \mathrm{y}+8.1731(0.0140) \mathrm{z}=6.1885(0.0155)$

Angle to previous plane (with approximate esd) $=8.325(0.117)$

* $0.0427(0.0013) \mathrm{Pd} 2 * 0.1010(0.0020) \mathrm{N} 2 *-0.1261(0.0020) \mathrm{C} 22 *-0.0908(0.0015) \mathrm{I} 1 * 0.0732(0.0015) \mathrm{I} 2$

Rms deviation of fitted atoms $=0.0911$

Refinement. The hydrogens atoms at NH were refined with DFIX, others as rigid.

Fractional atomic coordinates and isotropic or equivalent isotropic displacement parameters $\left(\AA^{2}\right)$

\begin{tabular}{|c|c|c|c|c|}
\hline & $x$ & $y$ & $z$ & $U_{\text {iso }} * / U_{\text {eq }}$ \\
\hline Pd1 & $0.47515(2)$ & $0.70685(3)$ & $1.00161(2)$ & $0.02890(9)$ \\
\hline $\mathrm{Pd} 2$ & $0.34849(2)$ & $0.47810(3)$ & $0.87459(2)$ & $0.02862(9)$ \\
\hline I1 & $0.30166(2)$ & $0.58520(3)$ & $0.99762(2)$ & $0.03447(9)$ \\
\hline I2 & $0.49727(2)$ & $0.62609(3)$ & $0.87564(2)$ & $0.03242(9)$ \\
\hline N1 & $0.4641(3)$ & $0.7879(4)$ & $1.1013(2)$ & $0.0380(9)$ \\
\hline H01 & $0.494(4)$ & $0.729(5)$ & $1.127(2)$ & $0.045(16)^{*}$ \\
\hline $\mathrm{N} 2$ & $0.2317(3)$ & $0.3510(4)$ & $0.8740(2)$ & $0.0370(9)$ \\
\hline H02 & $0.231(4)$ & $0.317(6)$ & $0.9151(15)$ & $0.048(17)^{*}$ \\
\hline $\mathrm{C} 1$ & $0.5929(3)$ & $0.9138(4)$ & $1.0531(2)$ & $0.0332(9)$ \\
\hline $\mathrm{C} 2$ & $0.5942(3)$ & $0.8107(4)$ & $1.0039(2)$ & $0.0286(9)$ \\
\hline $\mathrm{C} 3$ & $0.6740(3)$ & $0.7940(4)$ & $0.9634(2)$ & $0.0327(9)$ \\
\hline H3 & 0.677255 & 0.722141 & 0.931399 & $0.039^{*}$ \\
\hline $\mathrm{C} 4$ & $0.7488(4)$ & $0.8826(5)$ & $0.9699(3)$ & $0.0406(11)$ \\
\hline $\mathrm{H} 4$ & 0.803004 & 0.870965 & 0.942083 & $0.049^{*}$ \\
\hline $\mathrm{C} 5$ & $0.7454(4)$ & $0.9875(6)$ & $1.0164(3)$ & $0.0513(14)$ \\
\hline H5 & 0.796102 & 1.049342 & 1.019104 & $0.062^{*}$ \\
\hline C6 & $0.6688(4)$ & $1.0024(5)$ & $1.0585(3)$ & $0.0437(12)$ \\
\hline H6 & 0.667352 & 1.072824 & 1.091399 & $0.052 *$ \\
\hline $\mathrm{C} 7$ & $0.5084(4)$ & $0.9232(5)$ & $1.0990(2)$ & $0.0364(10)$ \\
\hline H7A & 0.527604 & 0.951407 & 1.145846 & $0.044^{*}$ \\
\hline H7B & 0.463367 & 0.989848 & 1.080537 & $0.044^{*}$ \\
\hline $\mathrm{C} 8$ & $0.3699(4)$ & $0.7860(6)$ & $1.1331(3)$ & 0.0505 (13) \\
\hline $\mathrm{H} 8 \mathrm{~A}$ & 0.347701 & 0.691992 & 1.134865 & $0.061^{*}$ \\
\hline H8B & 0.326034 & 0.836288 & 1.103039 & $0.061^{*}$ \\
\hline $\mathrm{C} 11$ & $0.3647(3)$ & $0.8443(5)$ & $1.2049(3)$ & $0.0426(11)$ \\
\hline $\mathrm{C} 12$ & $0.3065(4)$ & $0.9528(6)$ & $1.2173(4)$ & $0.0653(18)$ \\
\hline H12 & 0.275421 & 0.996211 & 1.180096 & $0.078^{*}$ \\
\hline $\mathrm{C} 13$ & $0.2939(5)$ & $0.9979(8)$ & $1.2842(4)$ & $0.083(2)$ \\
\hline H13 & 0.252076 & 1.070199 & 1.292786 & $0.100^{*}$ \\
\hline $\mathrm{C} 14$ & $0.3408(5)$ & $0.9395(8)$ & $1.3378(4)$ & 0.0750 (19) \\
\hline H14 & 0.332063 & 0.971284 & 1.383422 & $0.090^{*}$ \\
\hline
\end{tabular}




$\begin{array}{lllll}\text { C15 } & 0.4000(6) & 0.8356(8) & 1.3252(3) & 0.081(2) \\ \text { H15 } & 0.434133 & 0.795865 & 1.362036 & 0.097^{*} \\ \text { C16 } & 0.4105(5) & 0.7881(7) & 1.2594(3) & 0.0683(19) \\ \text { H16 } & 0.450879 & 0.713792 & 1.251593 & 0.082^{*} \\ \text { C21 } & 0.3024(3) & 0.3022(4) & 0.7638(2) & 0.0338(9) \\ \text { C22 } & 0.3629(3) & 0.4095(4) & 0.7789(2) & 0.0294(9) \\ \text { C23 } & 0.4149(3) & 0.4648(5) & 0.7258(2) & 0.0345(10) \\ \text { H23 } & 0.455206 & 0.538695 & 0.734839 & 0.041^{*} \\ \text { C24 } & 0.4089(4) & 0.4137(5) & 0.6598(3) & 0.0412(11) \\ \text { H24 } & 0.445559 & 0.451778 & 0.623915 & 0.049^{*} \\ \text { C25 } & 0.3493(4) & 0.3067(5) & 0.6458(3) & 0.0431(11) \\ \text { H25 } & 0.345393 & 0.271395 & 0.600469 & 0.052^{*} \\ \text { C26 } & 0.2962(3) & 0.2519(5) & 0.6974(3) & 0.0384(10) \\ \text { H26 } & 0.254951 & 0.179341 & 0.687572 & 0.046^{*} \\ \text { C27 } & 0.2478(4) & 0.2432(5) & 0.8225(3) & 0.0397(11) \\ \text { H27A } & 0.186886 & 0.207751 & 0.805648 & 0.048^{*} \\ \text { H27B } & 0.283548 & 0.168638 & 0.843681 & 0.048^{*} \\ \text { C28 } & 0.1430(3) & 0.4257(5) & 0.8621(3) & 0.0406(11) \\ \text { H28A } & 0.141848 & 0.459322 & 0.814148 & 0.049^{*} \\ \text { H28B } & 0.141125 & 0.504176 & 0.893271 & 0.049^{*} \\ \text { C31 } & 0.0568(4) & 0.3397(5) & 0.8743(3) & 0.0424(11) \\ \text { C32 } & 0.0347(4) & 0.3003(7) & 0.9398(3) & 0.0618(17) \\ \text { H32 } & 0.072487 & 0.328740 & 0.977520 & 0.074^{*} \\ \text { C33 } & -0.0430(5) & 0.2189(8) & 0.9513(4) & 0.076(2) \\ \text { H33 } & -0.057735 & 0.190253 & 0.996600 & 0.091^{*} \\ \text { C34 } & -0.0981(4) & 0.1800(7) & 0.8969(3) & 0.0585(15) \\ \text { H34 } & -0.151242 & 0.124423 & 0.904654 & 0.070^{*} \\ \text { C35 } & -0.0775(4) & 0.2200(6) & 0.8331(3) & 0.0485(13) \\ \text { H35 } & -0.116842 & 0.193593 & 0.795804 & 0.058^{*} \\ \text { C36 } & 0.0005(3) & 0.2995(5) & 0.8205(3) & 0.0442(12) \\ \text { H36 } & 0.014989 & 0.326193 & 0.774793 & 0.053^{*} \\ & & & & \end{array}$

Atomic displacement parameters $\left(\AA^{2}\right)$

\begin{tabular}{lllllll}
\hline & $U^{11}$ & $U^{22}$ & $U^{33}$ & $U^{12}$ & $U^{13}$ & $U^{23}$ \\
\hline Pd1 & $0.02632(17)$ & $0.02705(17)$ & $0.03331(18)$ & $-0.00158(13)$ & $-0.00086(13)$ & $-0.00183(13)$ \\
Pd2 & $0.02629(17)$ & $0.02353(17)$ & $0.03602(18)$ & $-0.00306(12)$ & $-0.00117(13)$ & $0.00140(13)$ \\
I1 & $0.02959(16)$ & $0.03506(17)$ & $0.03877(17)$ & $-0.00318(12)$ & $0.00233(12)$ & $0.00046(12)$ \\
I2 & $0.03033(16)$ & $0.03417(16)$ & $0.03276(16)$ & $-0.00964(11)$ & $0.00015(11)$ & $-0.00282(12)$ \\
N1 & $0.034(2)$ & $0.039(2)$ & $0.041(2)$ & $-0.0039(17)$ & $0.0036(17)$ & $-0.0084(18)$ \\
N2 & $0.035(2)$ & $0.032(2)$ & $0.044(2)$ & $-0.0045(17)$ & $0.0042(18)$ & $0.0047(18)$ \\
C1 & $0.044(3)$ & $0.027(2)$ & $0.029(2)$ & $-0.0038(19)$ & $-0.0059(18)$ & $0.0070(17)$ \\
C2 & $0.036(2)$ & $0.024(2)$ & $0.026(2)$ & $-0.0005(17)$ & $-0.0055(16)$ & $0.0069(16)$ \\
C3 & $0.034(2)$ & $0.025(2)$ & $0.039(2)$ & $-0.0056(17)$ & $-0.0025(18)$ & $0.0040(18)$ \\
C4 & $0.040(3)$ & $0.043(3)$ & $0.039(3)$ & $-0.016(2)$ & $0.002(2)$ & $0.006(2)$ \\
C5 & $0.063(3)$ & $0.050(3)$ & $0.041(3)$ & $-0.034(3)$ & $0.001(2)$ & $0.003(2)$ \\
C6 & $0.065(3)$ & $0.034(3)$ & $0.033(2)$ & $-0.020(2)$ & $-0.003(2)$ & $0.0038(19)$ \\
C7 & $0.050(3)$ & $0.027(2)$ & $0.032(2)$ & $0.000(2)$ & $-0.003(2)$ & $-0.0013(18)$
\end{tabular}




\begin{tabular}{lllllll} 
C8 & $0.038(3)$ & $0.057(3)$ & $0.056(3)$ & $-0.001(2)$ & $0.008(2)$ & $-0.010(3)$ \\
C11 & $0.035(3)$ & $0.041(3)$ & $0.052(3)$ & $-0.004(2)$ & $0.008(2)$ & $-0.008(2)$ \\
C12 & $0.045(3)$ & $0.064(4)$ & $0.087(4)$ & $0.014(3)$ & $-0.019(3)$ & $-0.025(3)$ \\
C13 & $0.052(4)$ & $0.085(5)$ & $0.113(5)$ & $0.021(3)$ & $-0.006(4)$ & $-0.057(4)$ \\
C14 & $0.062(4)$ & $0.093(5)$ & $0.070(4)$ & $-0.015(4)$ & $0.030(3)$ & $-0.025(4)$ \\
C15 & $0.094(6)$ & $0.101(6)$ & $0.047(3)$ & $0.016(4)$ & $0.022(4)$ & $0.011(4)$ \\
C16 & $0.087(5)$ & $0.067(4)$ & $0.051(3)$ & $0.033(4)$ & $0.019(3)$ & $0.012(3)$ \\
C21 & $0.030(2)$ & $0.025(2)$ & $0.046(2)$ & $0.0001(17)$ & $-0.0045(19)$ & $0.0013(19)$ \\
C22 & $0.029(2)$ & $0.023(2)$ & $0.036(2)$ & $0.0022(16)$ & $-0.0049(17)$ & $0.0008(17)$ \\
C23 & $0.036(2)$ & $0.027(2)$ & $0.041(2)$ & $-0.0030(18)$ & $-0.0046(19)$ & $0.0029(19)$ \\
C24 & $0.050(3)$ & $0.036(3)$ & $0.038(2)$ & $-0.003(2)$ & $-0.005(2)$ & $0.005(2)$ \\
C25 & $0.050(3)$ & $0.039(3)$ & $0.040(3)$ & $0.000(2)$ & $-0.009(2)$ & $-0.004(2)$ \\
C26 & $0.037(2)$ & $0.028(2)$ & $0.051(3)$ & $-0.0006(19)$ & $-0.010(2)$ & $-0.006(2)$ \\
C27 & $0.036(2)$ & $0.030(2)$ & $0.053(3)$ & $-0.0081(19)$ & $-0.002(2)$ & $-0.001(2)$ \\
C28 & $0.033(2)$ & $0.038(3)$ & $0.051(3)$ & $-0.0006(19)$ & $-0.002(2)$ & $-0.001(2)$ \\
C31 & $0.035(2)$ & $0.041(3)$ & $0.052(3)$ & $0.001(2)$ & $0.008(2)$ & $-0.005(2)$ \\
C32 & $0.043(3)$ & $0.092(5)$ & $0.051(3)$ & $-0.014(3)$ & $0.002(3)$ & $0.000(3)$ \\
C33 & $0.052(4)$ & $0.118(6)$ & $0.058(3)$ & $-0.022(4)$ & $0.019(3)$ & $0.008(4)$ \\
C34 & $0.032(3)$ & $0.071(4)$ & $0.073(4)$ & $-0.010(3)$ & $0.011(3)$ & $-0.004(3)$ \\
C35 & $0.030(2)$ & $0.046(3)$ & $0.069(3)$ & $-0.002(2)$ & $0.005(2)$ & $-0.006(3)$ \\
C36 & $0.032(2)$ & $0.043(3)$ & $0.057(3)$ & $0.001(2)$ & $0.001(2)$ & $-0.004(2)$ \\
& & & & & & \\
\hline
\end{tabular}

Geometric parameters $\left(\AA,{ }^{\circ}\right)$

\begin{tabular}{llll}
\hline $\mathrm{Pd} 1-\mathrm{C} 2$ & $1.986(5)$ & $\mathrm{C} 13-\mathrm{C} 14$ & $1.364(9)$ \\
$\mathrm{Pd} 1-\mathrm{N} 1$ & $2.104(4)$ & $\mathrm{C} 13-\mathrm{H} 13$ & 0.9500 \\
$\mathrm{Pd} 1-\mathrm{I} 2$ & $2.5959(5)$ & $\mathrm{C} 14-\mathrm{C} 15$ & $1.358(9)$ \\
$\mathrm{Pd} 1-\mathrm{I} 1$ & $2.7504(5)$ & $\mathrm{C} 14-\mathrm{H} 14$ & 0.9500 \\
$\mathrm{Pd} 2-\mathrm{C} 22$ & $1.991(4)$ & $\mathrm{C} 15-\mathrm{C} 16$ & $1.373(8)$ \\
$\mathrm{Pd} 2-\mathrm{N} 2$ & $2.090(4)$ & $\mathrm{C} 15-\mathrm{H} 15$ & 0.9500 \\
$\mathrm{Pd} 2-\mathrm{I} 2$ & $2.5801(4)$ & $\mathrm{C} 16-\mathrm{H} 16$ & 0.9500 \\
$\mathrm{Pd} 2-\mathrm{I} 1$ & $2.7030(5)$ & $\mathrm{C} 21-\mathrm{C} 26$ & $1.387(6)$ \\
$\mathrm{N} 1-\mathrm{C} 8$ & $1.477(7)$ & $\mathrm{C} 21-\mathrm{C} 22$ & $1.404(6)$ \\
$\mathrm{N} 1-\mathrm{C} 7$ & $1.491(6)$ & $\mathrm{C} 21-\mathrm{C} 27$ & $1.502(7)$ \\
$\mathrm{N} 1-\mathrm{H} 01$ & $0.884(19)$ & $\mathrm{C} 22-\mathrm{C} 23$ & $1.387(6)$ \\
$\mathrm{N} 2-\mathrm{C} 28$ & $1.482(6)$ & $\mathrm{C} 23-\mathrm{C} 24$ & $1.382(6)$ \\
$\mathrm{N} 2-\mathrm{C} 27$ & $1.487(7)$ & $\mathrm{C} 23-\mathrm{H} 23$ & 0.9500 \\
$\mathrm{~N} 2-\mathrm{H} 02$ & $0.87(2)$ & $\mathrm{C} 24-\mathrm{C} 25$ & $1.390(7)$ \\
$\mathrm{C} 1-\mathrm{C} 6$ & $1.399(7)$ & $\mathrm{C} 24-\mathrm{H} 24$ & 0.9500 \\
$\mathrm{C} 1-\mathrm{C} 2$ & $1.404(6)$ & $\mathrm{C} 25-\mathrm{C} 26$ & $1.370(7)$ \\
$\mathrm{C} 1-\mathrm{C} 7$ & $1.501(7)$ & $\mathrm{C} 25-\mathrm{H} 25$ & 0.9500 \\
$\mathrm{C} 2-\mathrm{C} 3$ & $1.392(7)$ & $\mathrm{C} 26-\mathrm{H} 26$ & 0.9500 \\
$\mathrm{C} 3-\mathrm{C} 4$ & $1.388(6)$ & $\mathrm{C} 27-\mathrm{H} 27 \mathrm{~A}$ & 0.9900 \\
$\mathrm{C} 3-\mathrm{H} 3$ & 0.9500 & $\mathrm{C} 27-\mathrm{H} 27 \mathrm{~B}$ & 0.9900 \\
$\mathrm{C} 4-\mathrm{C} 5$ & $1.383(8)$ & $\mathrm{C} 28-\mathrm{C} 31$ & $1.516(7)$ \\
$\mathrm{C} 4-\mathrm{H} 4$ & 0.9500 & $\mathrm{C} 28-\mathrm{H} 28 \mathrm{~A}$ & 0.9900 \\
$\mathrm{C} 5-\mathrm{C} 6$ & $1.373(8)$ & $\mathrm{C} 28-\mathrm{H} 28 \mathrm{~B}$ & 0.9900 \\
$\mathrm{C} 5-\mathrm{H} 5$ & 0.9500 & $\mathrm{C} 31-\mathrm{C} 32$ & $1.369(7)$ \\
& & &
\end{tabular}




\begin{tabular}{|c|c|c|c|}
\hline $\mathrm{C} 6-\mathrm{H} 6$ & 0.9500 & $\mathrm{C} 31-\mathrm{C} 36$ & $1.375(7)$ \\
\hline C7-H7A & 0.9900 & $\mathrm{C} 32-\mathrm{C} 33$ & $1.390(8)$ \\
\hline C7-H7B & 0.9900 & $\mathrm{C} 32-\mathrm{H} 32$ & 0.9500 \\
\hline $\mathrm{C} 8-\mathrm{C} 11$ & $1.512(8)$ & $\mathrm{C} 33-\mathrm{C} 34$ & $1.368(8)$ \\
\hline $\mathrm{C} 8-\mathrm{H} 8 \mathrm{~A}$ & 0.9900 & $\mathrm{C} 33-\mathrm{H} 33$ & 0.9500 \\
\hline $\mathrm{C} 8-\mathrm{H} 8 \mathrm{~B}$ & 0.9900 & $\mathrm{C} 34-\mathrm{C} 35$ & $1.336(8)$ \\
\hline $\mathrm{C} 11-\mathrm{C} 16$ & $1.361(7)$ & $\mathrm{C} 34-\mathrm{H} 34$ & 0.9500 \\
\hline $\mathrm{C} 11-\mathrm{C} 12$ & $1.384(7)$ & $\mathrm{C} 35-\mathrm{C} 36$ & $1.386(7)$ \\
\hline $\mathrm{C} 12-\mathrm{C} 13$ & $1.388(9)$ & C35-H35 & 0.9500 \\
\hline $\mathrm{C} 12-\mathrm{H} 12$ & 0.9500 & $\mathrm{C} 36-\mathrm{H} 36$ & 0.9500 \\
\hline $\mathrm{C} 2-\mathrm{Pd} 1-\mathrm{N} 1$ & $81.12(17)$ & $\mathrm{C} 13-\mathrm{C} 12-\mathrm{H} 12$ & 120.1 \\
\hline $\mathrm{C} 2-\mathrm{Pd} 1-\mathrm{I} 2$ & $94.40(13)$ & $\mathrm{C} 14-\mathrm{C} 13-\mathrm{C} 12$ & $120.7(6)$ \\
\hline $\mathrm{N} 1-\mathrm{Pd} 1-\mathrm{I} 2$ & $174.83(12)$ & $\mathrm{C} 14-\mathrm{C} 13-\mathrm{H} 13$ & 119.6 \\
\hline $\mathrm{C} 2-\mathrm{Pd} 1-\mathrm{I} 1$ & $174.71(12)$ & $\mathrm{C} 12-\mathrm{C} 13-\mathrm{H} 13$ & 119.6 \\
\hline $\mathrm{N} 1-\mathrm{Pd} 1-\mathrm{I} 1$ & $97.21(11)$ & $\mathrm{C} 15-\mathrm{C} 14-\mathrm{C} 13$ & $119.4(6)$ \\
\hline $\mathrm{I} 2-\mathrm{Pd} 1-\mathrm{I} 1$ & $87.023(13)$ & $\mathrm{C} 15-\mathrm{C} 14-\mathrm{H} 14$ & 120.3 \\
\hline $\mathrm{C} 22-\mathrm{Pd} 2-\mathrm{N} 2$ & $82.55(17)$ & $\mathrm{C} 13-\mathrm{C} 14-\mathrm{H} 14$ & 120.3 \\
\hline $\mathrm{C} 22-\mathrm{Pd} 2-\mathrm{I} 2$ & $96.72(12)$ & $\mathrm{C} 14-\mathrm{C} 15-\mathrm{C} 16$ & $120.1(7)$ \\
\hline $\mathrm{N} 2-\mathrm{Pd} 2-\mathrm{I} 2$ & $177.54(12)$ & $\mathrm{C} 14-\mathrm{C} 15-\mathrm{H} 15$ & 120.0 \\
\hline $\mathrm{C} 22-\mathrm{Pd} 2-\mathrm{I} 1$ & $170.83(12)$ & $\mathrm{C} 16-\mathrm{C} 15-\mathrm{H} 15$ & 120.0 \\
\hline $\mathrm{N} 2-\mathrm{Pd} 2-\mathrm{I} 1$ & $92.69(12)$ & $\mathrm{C} 11-\mathrm{C} 16-\mathrm{C} 15$ & $121.9(6)$ \\
\hline $\mathrm{I} 2-\mathrm{Pd} 2-\mathrm{I} 1$ & $88.352(14)$ & $\mathrm{C} 11-\mathrm{C} 16-\mathrm{H} 16$ & 119.1 \\
\hline $\mathrm{Pd} 2-\mathrm{I} 1-\mathrm{Pd} 1$ & $88.605(13)$ & $\mathrm{C} 15-\mathrm{C} 16-\mathrm{H} 16$ & 119.1 \\
\hline $\mathrm{Pd} 2-\mathrm{I} 2-\mathrm{Pd} 1$ & $94.767(14)$ & $\mathrm{C} 26-\mathrm{C} 21-\mathrm{C} 22$ & $120.5(4)$ \\
\hline $\mathrm{C} 8-\mathrm{N} 1-\mathrm{C} 7$ & $114.1(4)$ & $\mathrm{C} 26-\mathrm{C} 21-\mathrm{C} 27$ & $122.2(4)$ \\
\hline $\mathrm{C} 8-\mathrm{N} 1-\mathrm{Pd} 1$ & $116.8(3)$ & $\mathrm{C} 22-\mathrm{C} 21-\mathrm{C} 27$ & $117.3(4)$ \\
\hline $\mathrm{C} 7-\mathrm{N} 1-\mathrm{Pd} 1$ & $106.7(3)$ & $\mathrm{C} 23-\mathrm{C} 22-\mathrm{C} 21$ & $118.4(4)$ \\
\hline $\mathrm{C} 8-\mathrm{N} 1-\mathrm{H} 01$ & $101(4)$ & $\mathrm{C} 23-\mathrm{C} 22-\mathrm{Pd} 2$ & 128.0 \\
\hline $\mathrm{C} 7-\mathrm{N} 1-\mathrm{H} 01$ & $114(4)$ & $\mathrm{C} 21-\mathrm{C} 22-\mathrm{Pd} 2$ & $113.1(3)$ \\
\hline $\mathrm{Pd} 1-\mathrm{N} 1-\mathrm{H} 01$ & $104(4)$ & $\mathrm{C} 24-\mathrm{C} 23-\mathrm{C} 22$ & $120.9(4)$ \\
\hline $\mathrm{C} 28-\mathrm{N} 2-\mathrm{C} 27$ & $113.1(4)$ & $\mathrm{C} 24-\mathrm{C} 23-\mathrm{H} 23$ & 119.6 \\
\hline $\mathrm{C} 28-\mathrm{N} 2-\mathrm{Pd} 2$ & $111.8(3)$ & $\mathrm{C} 22-\mathrm{C} 23-\mathrm{H} 23$ & 119.6 \\
\hline $\mathrm{C} 27-\mathrm{N} 2-\mathrm{Pd} 2$ & $108.6(3)$ & $\mathrm{C} 23-\mathrm{C} 24-\mathrm{C} 25$ & $120.0(5)$ \\
\hline $\mathrm{C} 28-\mathrm{N} 2-\mathrm{H} 02$ & $109(4)$ & $\mathrm{C} 23-\mathrm{C} 24-\mathrm{H} 24$ & 120.0 \\
\hline $\mathrm{C} 27-\mathrm{N} 2-\mathrm{H} 02$ & $110(4)$ & $\mathrm{C} 25-\mathrm{C} 24-\mathrm{H} 24$ & 120.0 \\
\hline $\mathrm{Pd} 2-\mathrm{N} 2-\mathrm{H} 02$ & $104(4)$ & $\mathrm{C} 26-\mathrm{C} 25-\mathrm{C} 24$ & $120.2(5)$ \\
\hline $\mathrm{C} 6-\mathrm{C} 1-\mathrm{C} 2$ & $120.1(5)$ & $\mathrm{C} 26-\mathrm{C} 25-\mathrm{H} 25$ & 119.9 \\
\hline $\mathrm{C} 6-\mathrm{C} 1-\mathrm{C} 7$ & $122.3(4)$ & $\mathrm{C} 24-\mathrm{C} 25-\mathrm{H} 25$ & 119.9 \\
\hline $\mathrm{C} 2-\mathrm{C} 1-\mathrm{C} 7$ & $117.6(4)$ & $\mathrm{C} 25-\mathrm{C} 26-\mathrm{C} 21$ & $120.1(4)$ \\
\hline $\mathrm{C} 3-\mathrm{C} 2-\mathrm{C} 1$ & $119.0(4)$ & $\mathrm{C} 25-\mathrm{C} 26-\mathrm{H} 26$ & 120.0 \\
\hline $\mathrm{C} 3-\mathrm{C} 2-\mathrm{Pd} 1$ & $128.4(3)$ & $\mathrm{C} 21-\mathrm{C} 26-\mathrm{H} 26$ & 120.0 \\
\hline $\mathrm{C} 1-\mathrm{C} 2-\mathrm{Pd} 1$ & $112.6(3)$ & $\mathrm{N} 2-\mathrm{C} 27-\mathrm{C} 21$ & $107.9(4)$ \\
\hline $\mathrm{C} 4-\mathrm{C} 3-\mathrm{C} 2$ & $119.8(5)$ & $\mathrm{N} 2-\mathrm{C} 27-\mathrm{H} 27 \mathrm{~A}$ & 110.1 \\
\hline $\mathrm{C} 4-\mathrm{C} 3-\mathrm{H} 3$ & 120.1 & $\mathrm{C} 21-\mathrm{C} 27-\mathrm{H} 27 \mathrm{~A}$ & 110.1 \\
\hline $\mathrm{C} 2-\mathrm{C} 3-\mathrm{H} 3$ & 120.1 & $\mathrm{~N} 2-\mathrm{C} 27-\mathrm{H} 27 \mathrm{~B}$ & 110.1 \\
\hline $\mathrm{C} 5-\mathrm{C} 4-\mathrm{C} 3$ & $120.9(5)$ & $\mathrm{C} 21-\mathrm{C} 27-\mathrm{H} 27 \mathrm{~B}$ & 110.1 \\
\hline $\mathrm{C} 5-\mathrm{C} 4-\mathrm{H} 4$ & 119.6 & $\mathrm{H} 27 \mathrm{~A}-\mathrm{C} 27-\mathrm{H} 27 \mathrm{~B}$ & 108.4 \\
\hline
\end{tabular}




\begin{tabular}{|c|c|}
\hline $\mathrm{C} 3-\mathrm{C} 4-\mathrm{H} 4$ & 119.6 \\
\hline $\mathrm{C} 6-\mathrm{C} 5-\mathrm{C} 4$ & $120.0(5)$ \\
\hline $\mathrm{C} 6-\mathrm{C} 5-\mathrm{H} 5$ & 120.0 \\
\hline $\mathrm{C} 4-\mathrm{C} 5-\mathrm{H} 5$ & 120.0 \\
\hline $\mathrm{C} 5-\mathrm{C} 6-\mathrm{C} 1$ & 120.0 \\
\hline $\mathrm{C} 5-\mathrm{C} 6-\mathrm{H} 6$ & 120.0 \\
\hline $\mathrm{C} 1-\mathrm{C} 6-\mathrm{H} 6$ & 120.0 \\
\hline $\mathrm{N} 1-\mathrm{C} 7-\mathrm{C} 1$ & $107.5(4)$ \\
\hline $\mathrm{N} 1-\mathrm{C} 7-\mathrm{H} 7 \mathrm{~A}$ & 110.2 \\
\hline $\mathrm{C} 1-\mathrm{C} 7-\mathrm{H} 7 \mathrm{~A}$ & 110.2 \\
\hline $\mathrm{N} 1-\mathrm{C} 7-\mathrm{H} 7 \mathrm{~B}$ & 110.2 \\
\hline $\mathrm{C} 1-\mathrm{C} 7-\mathrm{H} 7 \mathrm{~B}$ & 110.2 \\
\hline $\mathrm{H} 7 \mathrm{~A}-\mathrm{C} 7-\mathrm{H} 7 \mathrm{~B}$ & 108.5 \\
\hline $\mathrm{N} 1-\mathrm{C} 8-\mathrm{C} 11$ & $115.4(5)$ \\
\hline $\mathrm{N} 1-\mathrm{C} 8-\mathrm{H} 8 \mathrm{~A}$ & 108.4 \\
\hline $\mathrm{C} 11-\mathrm{C} 8-\mathrm{H} 8 \mathrm{~A}$ & 108.4 \\
\hline $\mathrm{N} 1-\mathrm{C} 8-\mathrm{H} 8 \mathrm{~B}$ & 108.4 \\
\hline $\mathrm{C} 11-\mathrm{C} 8-\mathrm{H} 8 \mathrm{~B}$ & 108.4 \\
\hline $\mathrm{H} 8 \mathrm{~A}-\mathrm{C} 8-\mathrm{H} 8 \mathrm{~B}$ & 107.5 \\
\hline $\mathrm{C} 16-\mathrm{C} 11-\mathrm{C} 12$ & $118.2(5)$ \\
\hline $\mathrm{C} 16-\mathrm{C} 11-\mathrm{C} 8$ & $122.2(5)$ \\
\hline $\mathrm{C} 12-\mathrm{C} 11-\mathrm{C} 8$ & $119.5(5)$ \\
\hline $\mathrm{C} 11-\mathrm{C} 12-\mathrm{C} 13$ & $119.7(6)$ \\
\hline $\mathrm{C} 11-\mathrm{C} 12-\mathrm{H} 12$ & 120.1 \\
\hline $\mathrm{C} 6-\mathrm{C} 1-\mathrm{C} 2-\mathrm{C} 3$ & $3.4(6)$ \\
\hline $\mathrm{C} 7-\mathrm{C} 1-\mathrm{C} 2-\mathrm{C} 3$ & $-176.0(4)$ \\
\hline $\mathrm{C} 6-\mathrm{C} 1-\mathrm{C} 2-\mathrm{Pd} 1$ & $-175.9(4)$ \\
\hline $\mathrm{C} 7-\mathrm{C} 1-\mathrm{C} 2-\mathrm{Pd} 1$ & $4.7(5)$ \\
\hline $\mathrm{C} 1-\mathrm{C} 2-\mathrm{C} 3-\mathrm{C} 4$ & $-3.0(7)$ \\
\hline $\mathrm{Pd} 1-\mathrm{C} 2-\mathrm{C} 3-\mathrm{C} 4$ & $176.1(4)$ \\
\hline $\mathrm{C} 2-\mathrm{C} 3-\mathrm{C} 4-\mathrm{C} 5$ & $0.2(8)$ \\
\hline $\mathrm{C} 3-\mathrm{C} 4-\mathrm{C} 5-\mathrm{C} 6$ & $2.3(9)$ \\
\hline $\mathrm{C} 4-\mathrm{C} 5-\mathrm{C} 6-\mathrm{C} 1$ & $-1.9(8)$ \\
\hline $\mathrm{C} 2-\mathrm{C} 1-\mathrm{C} 6-\mathrm{C} 5$ & $-0.9(7)$ \\
\hline $\mathrm{C} 7-\mathrm{C} 1-\mathrm{C} 6-\mathrm{C} 5$ & $178.5(5)$ \\
\hline $\mathrm{C} 8-\mathrm{N} 1-\mathrm{C} 7-\mathrm{C} 1$ & $-168.6(4)$ \\
\hline $\mathrm{Pd} 1-\mathrm{N} 1-\mathrm{C} 7-\mathrm{C} 1$ & $-38.0(4)$ \\
\hline $\mathrm{C} 6-\mathrm{C} 1-\mathrm{C} 7-\mathrm{N} 1$ & $-155.9(4)$ \\
\hline $\mathrm{C} 2-\mathrm{C} 1-\mathrm{C} 7-\mathrm{N} 1$ & $23.5(6)$ \\
\hline $\mathrm{C} 7-\mathrm{N} 1-\mathrm{C} 8-\mathrm{C} 11$ & $-54.9(7)$ \\
\hline $\mathrm{Pd} 1-\mathrm{N} 1-\mathrm{C} 8-\mathrm{C} 11$ & $179.6(4)$ \\
\hline $\mathrm{N} 1-\mathrm{C} 8-\mathrm{C} 11-\mathrm{C} 16$ & $-64.5(8)$ \\
\hline $\mathrm{N} 1-\mathrm{C} 8-\mathrm{C} 11-\mathrm{C} 12$ & $120.1(6)$ \\
\hline $\mathrm{C} 16-\mathrm{C} 11-\mathrm{C} 12-\mathrm{C} 13$ & $-2.3(9)$ \\
\hline $\mathrm{C} 8-\mathrm{C} 11-\mathrm{C} 12-\mathrm{C} 13$ & $173.3(6)$ \\
\hline $\mathrm{C} 11-\mathrm{C} 12-\mathrm{C} 13-\mathrm{C} 14$ & $2.4(10)$ \\
\hline $\mathrm{C} 12-\mathrm{C} 13-\mathrm{C} 14-\mathrm{C} 15$ & $-0.4(12)$ \\
\hline
\end{tabular}

$\begin{array}{ll}\mathrm{N} 2-\mathrm{C} 28-\mathrm{C} 31 & 112.3(4) \\ \mathrm{N} 2-\mathrm{C} 28-\mathrm{H} 28 \mathrm{~A} & 109.1 \\ \mathrm{C} 31-\mathrm{C} 28-\mathrm{H} 28 \mathrm{~A} & 109.1 \\ \mathrm{~N} 2-\mathrm{C} 28-\mathrm{H} 28 \mathrm{~B} & 109.1 \\ \mathrm{C} 31-\mathrm{C} 28-\mathrm{H} 28 \mathrm{~B} & 109.1 \\ \mathrm{H} 28 \mathrm{~A}-\mathrm{C} 28-\mathrm{H} 28 \mathrm{~B} & 107.9 \\ \mathrm{C} 32-\mathrm{C} 31-\mathrm{C} 36 & 119.2(5) \\ \mathrm{C} 32-\mathrm{C} 31-\mathrm{C} 28 & 119.8(5) \\ \mathrm{C} 36-\mathrm{C} 31-\mathrm{C} 28 & 121.0(5) \\ \mathrm{C} 31-\mathrm{C} 32-\mathrm{C} 33 & 120.1(6) \\ \mathrm{C} 31-\mathrm{C} 32-\mathrm{H} 32 & 119.9 \\ \mathrm{C} 33-\mathrm{C} 32-\mathrm{H} 32 & 119.9 \\ \mathrm{C} 34-\mathrm{C} 33-\mathrm{C} 32 & 119.7(6) \\ \mathrm{C} 34-\mathrm{C} 33-\mathrm{H} 33 & 120.1\end{array}$

C34-C33-H33 120.1

$\mathrm{C} 32-\mathrm{C} 33-\mathrm{H} 33 \quad 120.1$

$\mathrm{C} 35-\mathrm{C} 34-\mathrm{C} 33 \quad 120.3$ (6)

$\mathrm{C} 35-\mathrm{C} 34-\mathrm{H} 34 \quad 119.8$

$\mathrm{C} 33-\mathrm{C} 34-\mathrm{H} 34 \quad 119.8$

$\mathrm{C} 34-\mathrm{C} 35-\mathrm{C} 36 \quad 120.8(6)$

$\mathrm{C} 34-\mathrm{C} 35-\mathrm{H} 35 \quad 119.6$

$\mathrm{C} 36-\mathrm{C} 35-\mathrm{H} 35$

$\mathrm{C} 31-\mathrm{C} 36-\mathrm{C} 35$

$\mathrm{C} 31-\mathrm{C} 36-\mathrm{H} 36 \quad 120.1$

$\mathrm{C} 35-\mathrm{C} 36-\mathrm{H} 36 \quad 120.1$

$\mathrm{C} 26-\mathrm{C} 21-\mathrm{C} 22-\mathrm{C} 23 \quad 0.9(7)$

$\mathrm{C} 27-\mathrm{C} 21-\mathrm{C} 22-\mathrm{C} 23 \quad 179.3$ (4)

$\mathrm{C} 26-\mathrm{C} 21-\mathrm{C} 22-\mathrm{Pd} 2 \quad 173.1$ (4)

$\mathrm{C} 27-\mathrm{C} 21-\mathrm{C} 22-\mathrm{Pd} 2 \quad-8.5(5)$

$\mathrm{C} 21-\mathrm{C} 22-\mathrm{C} 23-\mathrm{C} 24 \quad-1.3(7)$

$\mathrm{Pd} 2-\mathrm{C} 22-\mathrm{C} 23-\mathrm{C} 24 \quad-172.2(4)$

$\mathrm{C} 22-\mathrm{C} 23-\mathrm{C} 24-\mathrm{C} 25 \quad 0.8(8)$

$\mathrm{C} 23-\mathrm{C} 24-\mathrm{C} 25-\mathrm{C} 26 \quad 0.2(8)$

$\mathrm{C} 24-\mathrm{C} 25-\mathrm{C} 26-\mathrm{C} 21 \quad-0.7(8)$

$\mathrm{C} 22-\mathrm{C} 21-\mathrm{C} 26-\mathrm{C} 25 \quad 0.1$ (7)

$\mathrm{C} 27-\mathrm{C} 21-\mathrm{C} 26-\mathrm{C} 25 \quad-178.2(5)$

$\mathrm{C} 28-\mathrm{N} 2-\mathrm{C} 27-\mathrm{C} 21 \quad 90.4(5)$

$\mathrm{Pd} 2-\mathrm{N} 2-\mathrm{C} 27-\mathrm{C} 21 \quad-34.3(4)$

$\mathrm{C} 26-\mathrm{C} 21-\mathrm{C} 27-\mathrm{N} 2 \quad-152.4(4)$

$\mathrm{C} 22-\mathrm{C} 21-\mathrm{C} 27-\mathrm{N} 2 \quad 29.2(6)$

$\mathrm{C} 27-\mathrm{N} 2-\mathrm{C} 28-\mathrm{C} 31 \quad 67.4(5)$

$\mathrm{Pd} 2-\mathrm{N} 2-\mathrm{C} 28-\mathrm{C} 31 \quad-169.6$ (3)

$\mathrm{N} 2-\mathrm{C} 28-\mathrm{C} 31-\mathrm{C} 32 \quad 69.7(6)$

$\mathrm{N} 2-\mathrm{C} 28-\mathrm{C} 31-\mathrm{C} 36 \quad-109.8(6)$

$\mathrm{C} 36-\mathrm{C} 31-\mathrm{C} 32-\mathrm{C} 33 \quad 1.0(8)$

$\mathrm{C} 28-\mathrm{C} 31-\mathrm{C} 32-\mathrm{C} 33 \quad-178.5(6)$

$\mathrm{C} 31-\mathrm{C} 32-\mathrm{C} 33-\mathrm{C} 34-1.1(10)$

$\mathrm{C} 32-\mathrm{C} 33-\mathrm{C} 34-\mathrm{C} 35 \quad 0.1$ (11) 


$\begin{array}{ll}\mathrm{C} 13-\mathrm{C} 14-\mathrm{C} 15-\mathrm{C} 16 & -1.6(12) \\ \mathrm{C} 12-\mathrm{C} 11-\mathrm{C} 16-\mathrm{C} 15 & 0.3(11) \\ \mathrm{C} 8-\mathrm{C} 11-\mathrm{C} 16-\mathrm{C} 15 & -175.1(7) \\ \mathrm{C} 14-\mathrm{C} 15-\mathrm{C} 16-\mathrm{C} 11 & 1.7(13)\end{array}$

$\mathrm{C} 33-\mathrm{C} 34-\mathrm{C} 35-\mathrm{C} 36$
$\mathrm{C} 32-\mathrm{C} 31-\mathrm{C} 36-\mathrm{C} 35$
$\mathrm{C} 28-\mathrm{C} 31-\mathrm{C} 36-\mathrm{C} 35$
$\mathrm{C} 34-\mathrm{C} 35-\mathrm{C} 36-\mathrm{C} 31$

$1.0(10)$

$0.0(8)$

$179.5(5)$

$-1.0(9)$

Hydrogen-bond geometry $\left(A,{ }^{\circ}\right)$

\begin{tabular}{lllll}
\hline$D-\mathrm{H} \cdots A$ & $D-\mathrm{H}$ & $\mathrm{H} \cdots A$ & $D \cdots A$ & $D-\mathrm{H} \cdots A$ \\
\hline $\mathrm{C} 8-\mathrm{H} 8 A \cdots \mathrm{I} 1$ & 0.99 & 2.94 & $3.444(6)$ & 113 \\
\hline
\end{tabular}

